\title{
Towards the measurement of bonding electron densities in nanostructured materials
}

\author{
P.N.H. Nakashima ${ }^{1}$, X. Tan ${ }^{1}$, D. Peng ${ }^{2}$, A. Mortazavi ${ }^{3,4}$, L. Bourgeois ${ }^{1,5}$, D.R. Clarke ${ }^{3}$ \\ ${ }^{2}$ Department of Materials Science and Engineering, Monash University, Victoria 3800, Australia, ${ }^{2}$ Department of Physics, Norwegian \\ University of Science and Technology (NTNU), Trondheim, Norway; ${ }^{3}$ School of Engineering and Applied Sciences, Harvard \\ University, Cambridge, USA; ${ }^{4}$ Department of Physics, Chalmers University of Technology, Gothenburg, Sweden; ${ }^{5}$ Monash Centre for \\ Electron Microscopy, Monash University, Victoria 3800, Australia.
}

Philip.Nakashima@monash.edu

Quantitative convergent-beam electron diffraction (QCBED) has become established as a highly accurate and precise means of measuring bonding electrostatic potentials and electron densities [1]. To date, it has almost exclusively been performed using the Blochwave electron scattering formalism [2], which requires 3-dimensional periodicity throughout the scattering volume. This has restricted QCBED to bonding measurements in homogeneous, single-phased crystalline materials (just like X-ray diffraction).

The multislice formalism [3] for electron scattering dispenses with the requirement of periodicity in the direction of the electron beam. Furthermore, electron probe sizes are typically of order $1 \mathrm{~nm}$ in dimension for QCBED, rendering even very highly curved features in nanostructured materials locally planar relative to the electron probe. This means that nanostructures that share a crystallographically coherent interface with the surrounding matrix in which they are embedded, could in principle be analysed by multislice-based QCBED. Add to this the routine sub-nanometre precision in positioning electron probes in transmission electron microscopes, and there is the potential to map bonding charge density as a function of position in nanostructured materials for the first time.

We are attempting to measure bonding electron densities within a number of different nanostructures and also across their interfaces with the surrounding matrix material, using QCBED based on the multislice formalism. We will present some early results from several nanostructured materials such as those shown in Fig. 1 below.
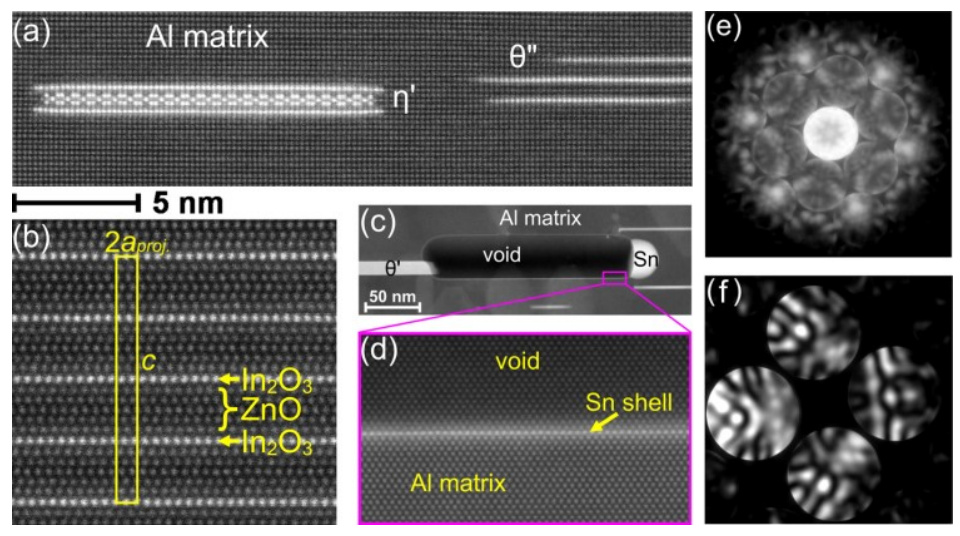

Figure 1. High angle annular dark field scanning transmission electron microscopy of an Al-Cu alloy [4] (a); a $\left(\mathrm{ZnO}_{\mathrm{k}} \mathrm{In}_{2} \mathrm{O}_{3}(\mathrm{k}=5)\right.$ thermoelectric oxide superlattice [5] (b); an Al-Cu-Sn alloy containing Sn-coated voids [6] (c \& d). The figure also presents a CBED pattern (e) from the material in part $\mathrm{b}$ and a CBED pattern (f) taken through a void like the one shown in parts $\mathrm{c}$ and $\mathrm{d}$.

[1] Nakashima, P. N. H., Smith, A. E., Etheridge, J. \& Muddle, B. C. (2011). Science 331, 1583.

[2] Bethe, H. A. (1928). Ann. Phys. (Berlin) 392, 55.

[3] Cowley, J. M. \& Moodie, A. F. (1957). Acta Cryst. 10, 609.

[4] Bourgeois, L., Zhang, Y., Zhang, Z., Chen, Y. \& Medhekar, N. V. (2020). Nature Commun. 11, 1248.

[5] Liang, X. \& Clarke, D. R. (2018). J. Appl. Phys. 124, 025101.

[6] Tan, X., Weyland, M., Chen, Y., Williams, T., Nakashima, P. N. H. \& Bourgeois, L. (2021). Acta Mater. 206, 116594.

\section{Keywords: Thermoelectric superlattice materials; alloys; electron diffraction; charge densities; chemical bonding}

We thank the Monash Centre for Electron Microscopy where all data were collected. Many thanks to A/Prof. Matthew Weyland and Prof. Joanne Etheridge for their expertise. We thank the Australian Research Council for funding (DP210100308).

Acta Cryst. (2021), A77, C279 